\title{
Constructal theory of pattern formation
}

\author{
A. Bejan \\ Duke University, Durham, North Carolina, USA \\ Received: 15 December 2005 - Published in Hydrol. Earth Syst. Sci. Discuss.: 19 July 2006 \\ Revised: 3 November 2006 - Accepted: 23 November 2006 - Published: 17 January 2007
}

\begin{abstract}
This review article shows that the occurrence of macroscopic flow configuration is a universal natural phenomenon that can be explained and predicted on the basis of a principle of physics (the constructal law): "For a flow system to persist in time (to survive) it must evolve in such a way that it provides easier and easier access to the currents that flow through it". The examples given in this article come from natural inanimate flow systems with configuration: duct cross-sections, open channel cross-sections, tree-shaped flow architectures, and turbulent flow structure (e.g., eddies, laminar lengths before transition). Other examples that are treated in the literature, and which support the constructal law, are the wedge-shape of turbulent shear layers, jets and plumes, the frequency of vortex shedding, Bénard convection in fluids and fluid-saturated porous media, dendritic solidification, the coalescence of solid parcels suspended in a flow, global atmospheric and oceanic circulation and climate, and virtually all architectural features of animal design. The constructal law stresses the importance of reserving a place for pure theory in research, and for constantly searching for new physics - new summarizing principles that are general, hence useful.
\end{abstract}

\section{The constructal law}

Why is geometry (shape, structure, similarity) a characteristic of natural flow systems? What is the basis for the hierarchy, complexity and rhythm of natural structures? Is there a single physics principle from which form and rhythm can be deduced, without any use of empiricism?

There is such a principle, and it is based on the common (universal) observation that if a flow system is endowed with sufficient freedom to change its configuration, then the system exhibits configurations that provide progressively better

Correspondence to: A. Bejan

(abejan@duke.edu) access routes for the currents that flow. Observations of this kind come in the billions, and they mean one thing: a time arrow is associated with the sequence of flow configurations that constitutes the existence of the system. Existing drawings are replaced by easier flowing drawings.

I formulated this principle in 1996 as the "constructal law" of the generation of flow configuration (Bejan, 1996, 1997ad):

"For a flow system to persist in time (to survive) it must evolve in such a way that it provides easier and easier access to the currents that flow through it".

This law is the basis for the "constructal theory" of the generation of flow configuration in nature, which was described in book form in Bejan (1997d). Today this entire body of work represents a new extension of thermodynamics: the thermodynamics of flow systems with configuration (Bejan, 2000; Bejan and Lorente, 2004, 2005; Lewins, 2003; Poirier, 2003; Rosa et al., 2004; Torre, 2004).

To see why the constructal law is a law of physics, ask why the constructal law is different than (i.e. distinct from, or complementary to) the other laws of thermodynamics. Think of an isolated thermodynamic system that is initially in a state of internal nonuniformity (e.g. regions of higher and lower pressures or temperature, separated by internal partitions that suddenly break). The first law and the second law account for billions of observations that describe a tendency in time, a "time arrow": if enough time passes, the isolated system settles into a state of equilibrium (no internal flows, maximum entropy at constant energy). The first law and second law speak of a black box. They say nothing about the configurations (the drawings) of the things that flow.

Classical thermodynamics was not concerned with the configurations of its nonequilibrium (flow) systems. It should have been. "The generation of flow configuration in time" is physics (a natural phenomenon) and it belongs in thermodynamics. 
This tendency, this time sequence of drawings that the flow system exhibits as it evolves, is the phenomenon covered by the constructal law. Not the drawings per se, but the time direction in which they morph if given freedom. No configuration in nature is "predetermined" or "destined" to be or to become a particular image. No one can say that the time sequence of configurations required by the constructal law should end with "this particular drawing". The actual evolution or lack of evolution (rigidity) of the drawing depends on many factors, which are mostly random, as we will see in Fig. 8. One cannot count on having the freedom to morph in peace (undisturbed).

The same can be said about the second law. No isolated system in nature is "predetermined" or "destined" to end up in a state of uniform intensive properties so that all future flows are ruled out. One cannot count on the removal of all the internal constraints. One can count even less on anything being left in peace, in isolation.

As a thought, the second law does proclaim the existence of a concept: the equilibrium in an isolated system, at sufficiently long times when all internal constraints have been removed. Likewise, the constructal law proclaims the existence of a concept: the "equilibrium flow architecture", which is defined as the configuration where all possibilities of increasing morphing freedom and flow access have been exhausted (Bejan and Lorente, 2004, 2005; Bejan, 2006).

Constructal theory is now a fast growing field with contributions from many sources, and with leads in many directions. This body of work has two main parts. The first is the focus of this review article: the use of the constructal law to predict and explain the occurrence of flow patterns in nature. The second part is the application of the constructal law as a scientific (physics) principle in engineering design. This activity of "design as science" is reviewed in Bejan et al. (2004), Bejan (2004, 2006) and Nield and Bejan (2006).

\section{Background}

Another way to delineate the place occupied by the constructal law in physics is by reviewing briefly some of the older and contemporary ideas that have been offered to shed light on the origin of flow configuration in nature. Extensive reviews of this body of thinking are provided in the first two books on the constructal law (Bejan, 1997d, 2000). In this section I focus only on the work that has emerged in geophysics, which is relevant in hydrology.

In brief, the development of science has shown that on numerous occasions scientists have considered as obvious the statement that "nature optimizes things". They based great discoveries on this intuitive feeling (from Heron of Alexandria and Pierre de Fermat in the propagation of light, to Paltridge, 1975, in global circulation and climate), and they did this "illegally" because a law of optimization (objective, final form) does not exist in physics.
This mental viewing was expressed in mathematical terms in the 1700s by the creators of variational calculus (Euler, Maupertuis, Leibnitz, Lagrange and others). Mathematics is the most powerful language in science, and language exists to facilitate and influence thinking. This is why the work that came after variational calculus has abandoned the search for optimal drawing (e.g. Heron, Fermat) and adopted instead the variational calculus paradigm: the search has been for the right global quantity (functional), which can be minimized or maximized by selecting the very special "optimal" function (the destined shape).

Ad-hoc invocations of "optimality" have been many, and their diversity is due to how one selected the system and the global quantity that was minimized or maximized. Two choices (classes) of ad-hoc optimality stand out:

MEP: entropy production, or maximum dissipation (e.g., Paltridge, 1975; North, 1981; Lin, 1982; Lorentz et al., 2001; Dewar, 2003).

EGM: Entropy generation minimization, or minimum pumping power, minimum work, minimum cost (e.g., Hess, 1913; Murray, 1926; Thompson, 1942; Bejan, 1982, 1996; Rodriguez-Iturbe and Rinaldo, 1997; Weibel, 2000).

All this ad-hoc work is important, taken by itself, or discussed along with the constructal law. It is important because it has been successful, over and over again. My earliest work was also of this kind, intuitive and ad-hoc: e.g., the prediction of transition to turbulence in all flow configurations by maximizing the rate of momentum transport (mixing) perpendicular to the shear flow (Bejan, 1982), and the prediction of the hair strand diameters and porosities of animal hair (fur) by minimizing the rate of body heat loss (Nield and Bejan, 1992; Bejan, 1993). Paltridge's work was preceded by the ad-hoc hypothesis of Malkus (1954), according to which the pattern of cells in Bénard convection is such that it maximizes the overall Nusselt number.

Ad-hoc invocations of an intuitively appealing idea did not make the idea universal enough to elevate it to the rank of law. The minimization of body heat loss is not the same as the maximization of mixing. The minimization of dissipation (EGM above) is even worse - it is the exact opposite of the maximization of dissipation (MEP above). At best, intuition is capricious, if not loaded with contradictions.

For science, the ad-hoc approaches have been divisive, not unifying. Maximum dissipation (MEP) appears to work in large-scale geophysical and other planetary flows. These are natural "inanimate" flow systems. Minimum dissipation (EGM), or maximum thermodynamic performance, is taken as obvious in animal design, engineering and social organization. These are "animate" flow systems. Why is there such disagreement between the animate and the inanimate? This should have been treated as a big question, after all, the animate and the inanimate obey all the laws of physics (e.g., $\mathrm{F}=\mathrm{ma}$ ). Lack of universality means that MEP and EGM are not laws of physics. 
Confusing the debate even more is the modern use of the word "entropy" to express the ad-hoc invocations of optimization in nature (note the E in MEP and EGM). Entropy is the thermodynamic property for which the second law serves as definition (in the same way that energy is the thermodynamic property defined based on the first law, and temperature is the property defined by the zeroth law; see e.g. Bejan, 1997d, chapters 1 and 2). The use of "entropy" in this discussion has perpetuated the view that, somehow, the second law accounts for the phenomenon of organization in nature. This is why we read that "order" can be derived from the second law (Swenson, 1989), that MEP can be deduced from existing principles (Dewar, 2003) and that "maximum entropy production is an organizational principle that potentially unifies biological and physical processes" (Dewar, quoted in Whitfield, 2005, which makes no sense because it is the opposite of what governs biological motors and our engines). And even if such claims were correct, then the derived statement (e.g. MEP) is at best a theorem, not a self-standing law.

Compared with the intuitive approaches reviewed above, the constructal law stands out in many important respects. The constructal law is not about a universal function, minimization, maximization, or optimal solution, and it is certainly not about entropy and the second law. The constructal law is about a previously overlooked phenomenon of all physics (the generation of flow configuration in time), and the time arrow of this phenomenon. The law is the universal observation that in time existing flow configurations are replaced by configurations that provide greater (easier, faster) access to the currents that flow.

Said another way, the constructal law is the statement that makes the time evolution of design (drawing) a principle of all physics. That I called it a law in 1996 was not a claim, but a proposal. Time will tell whether this proposal has merit, and time has been telling. Since 1996, more and more work is showing that the constructal law is in agreement with physical observations. Some of this work is reviewed here in Sects. 3-7, in Bejan (2006) and at http://www.constructal.org. Even more, when examined from the perspective of the constructal law, all the published success with ad-hoc intuitive statements such as MEP and EGM contributes enormous and independent support for the constuctal law. Everything now fits under one theoretical tent, all of design in nature, the animate and the inanimate, even the apparent contradiction between maximization of dissipation (MEP) and minimization of dissipation (EGM) (this most recent step of unification is explained in detail in the constructal theory of global circulation and climate reported by Reis and Bejan, 2006; see also Bejan, 2006).

The apparent overlap between the conceptual domain of constructal theory and optimality invocations is the source of the opposition expressed by three of the reviewers of this article. The fact is that the constructal law and ad-hoc optimality are two different mental viewings. An example of overlap is given in the last two paragraphs of Sect. 6. Another example is the constructal law of 1996 versus the model of West et al. (1997) consisting of dendritic flows, to account for allometric laws in animal design. Leaving aside the major difference between the two approaches (namely, modeling (making a copy/facsimile of nature) is empiricism, not theory), note that the model of West et al. is based on at least three ad hoc assumptions:

1. There is a "space-filling fractal-like branching pattern" (read: tree).

2. The final branch of the network is a size-invariant unit.

3. The energy required to distribute resources is minimized.

These three features were already present in 1996 constructal theory, not as convenient assumptions to polish a model and make it work but as invocations of a single principle: the constructal law. West and Brown (2005) acknowledged the overlap. Specifically, feature 3 is covered by the constructal law, feature 1 is the tree-flow architecture that in constructal theory is deduced from the constructal law, and feature 2 is the smallest-element scale that is fixed in all the constructal tree architectures. To repeat, in constructal theory the treeshaped flow is a discovery, not an observation and not an assumption.

Because features 1 to 3 are shared by constructal theory and by the model of West et al., every single allometric law that West et al. connect to their model is an affirmation of the validity of constructal theory. Every success of constructal theory in domains well beyond the reach of their model (e.g., river basins, flight, running, swimming, dendritic solidification, global circulation, mud cracks) is an indication that animal design is an integral part of a general theoretical framework - a new thermodynamics - that unites biology with physics and engineering.

\section{Natural flow configurations}

There are several classes of natural flow configurations, and each class can be derived from the constructal law in several ways: analytically (pencil \& paper), based on numerical simulations of morphing flow structures, approximately or more accurately, blindly (e.g. random search) or using strategy (shortcuts, memory), etc. How to deduce a class of flow configurations by invoking the constructal law is a thought that should not be confused with the constructal law. "How to deduce" is an expression of the researcher's freedom to choose the method of investigation (Bejan, 2004, p. 58). The constructal law statement is general: it does not use words such as tree, complex vs. simple, optimal vs. suboptimal, and natural vs. engineered.

Classes of flow configurations that our group (at Duke and abroad) has treated in detail are duct cross-sectional 


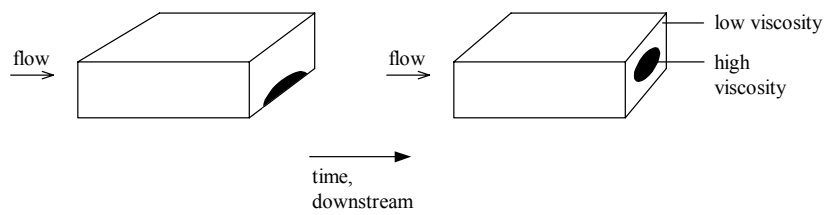

Fig. 1. The evolution of the cross-sectional configuration of a stream composed of two liquids, low viscosity and high viscosity. In time, the low viscosity liquid coats all the walls, and the high viscosity liquid migrates toward the center. This tendency of "selflubrication" is the action of the constructal law of the generation of flow configuration in geophysics (e.g. volcanic discharges, drawn after Carrigan, 1994) and in many biological systems.

shapes, river cross-sectional shapes, internal spacings, turbulent flow structure, animal movement, physiological on and off flows, tree-shaped architectures, dendritic solidification (snowflakes), Bénard convection and global circulation and climate. In this paper I review some of the main features and theoretical conclusions. More detailed accounts of these results and the body of literature that preceded it was given in my books (Bejan, 1997d, 2000, 2006).

\section{Duct cross-sections}

Blood vessels and pulmonary airways have round crosssections. Subterranean rivers, volcanic discharges, earth worms and ants carve galleries that have round crosssections. These many phenomena of flow configuration generation have been reasoned (Bejan, 1997d) by invoking the constructal law for the individual duct, or for the flow system $(\Sigma)$ that incorporates the duct. If the duct has a finite size (fixed cross-sectional area $A$ ) and the freedom to change its cross-sectional shape, then, in time, the shape will evolve such that the stream that flows through the duct flows with less resistance. If the system $(\Sigma)$ is isolated and consists of the duct and the two pressure reservoirs connected to the ends of the duct, then the duct architecture will evolve such that the entire system reaches equilibrium (no flow, uniform pressure) faster.

The duct cross-section evolves in time toward the round shape. This evolution cannot be witnessed in blood vessels and bronchial passages because our observation time scale (lifetime) is too short in comparison with the time scale of the evolution of a living system. The morphing of a round gallery can be observed during erosion processes in soil, following a sudden rainfall. It can be observed in the evolution of a volcanic lava conduit, where lava with lower viscosity coats the wall of the conduit, and lava with higher viscosity positions itself near the central part of the cross-section (Carrigan and Eichelberger, 1990; Carrigan, 1994). To have it the other way - high viscosity on the periphery and low viscosity in the center - would be a violation of the constructal law.
Additional support for the constructal law is provided by laboratory simulations of lava flow with high-viscosity intrusions (Fig. 1). Initially, the intrusion has a flat crosssection, and is positioned near the wall of the conduit. In time, i.e. downstream, the intrusion not only migrates toward the center of the cross-section but also develops a round cross-section of its own.

This tendency matches what is universally observed when a jet (laminar or turbulent) is injected into a fluid reservoir. If the jet initially has a flat cross-section, then further downstream it develops into one or more thicker jets with round cross-sections. The opposite trend is not observed: a round jet does not evolve into a flat jet.

The superiority of the round shape relative to other shapes is an important aspect the generalization of which has become a new addition to the thermodynamics of nonequilibrium systems: the "thermodynamics of systems with configuration" (Bejan and Lorente, 2004, 2005; Bejan, 2006).

For example, if the duct is straight and the perimeter of the fixed- $A$ cross-section is $p$ (variable), then the pressure drop $(\Delta P)$ per unit length $(\Delta L)$ is $\Delta P / \Delta L=\left(2 f / D_{h}\right) \rho V^{2}$, where $D_{h}=4 A / p, V$ is the mean fluid velocity $(\dot{m} / \rho A$, fixed) and $\mathrm{f}$ is the friction factor. If the flow regime is laminar and fully developed, then $f=\mathrm{Po} / \mathrm{Re}$, where $\mathrm{Re}=D_{h} V / \nu$, the kinematic viscosity is $v$, and Po is a factor that depends solely on the shape of the cross-section. For example, $\mathrm{Po}=16$ for a round cross-section with Poiseuille flow through it. For a very flat rectangular cross-section, Po is 24 . The duct flow resistance is

$$
\frac{\Delta P / \Delta L}{\dot{m}}=\frac{v}{8 A^{2}}\left(\operatorname{Po} \frac{p^{2}}{A}\right)
$$

where the group in parentheses depends only on the shape of the cross-section. This group governs the morphing direction in time.

Table 1 shows the values of the group ( $\mathrm{Po} p^{2} / A$ ) for several regular polygonal cross sections. Even though the round shape is the best, the nearly round shapes perform almost as well. For example, the relative change in $p^{2} \mathrm{Po} / A$ from the hexagon to the circle is only 3.7 percent. Square ducts have a flow resistance that is only 9.1 percent greater than that of hexagonal ducts.

Even if the duct cross-section is imperfect - that is, with features such as angles between flat spots, which concentrate fluid friction - its performance is nearly as good as it can be. Diversity (several near-optimal shapes) goes with the constructal law, not against it. Furthermore, the ceiling of performance of all the possible cross-sections can be predicted quite accurately when the global constraints $(A, \dot{m})$ are specified. 
Table 1. The laminar flow resistances of ducts with regular polygonal cross sections with $\mathrm{n}$ sides (Bejan, 1997d).

\begin{tabular}{llll}
\hline$n$ & Po & $p / A^{1 / 2}$ & $p^{2} \mathrm{Po} / A$ \\
\hline 3 & 13.33 & 4.559 & 277.1 \\
4 & 14.23 & 4 & 227.6 \\
6 & 15.054 & 3.722 & 208.6 \\
8 & 15.412 & 3.641 & 204.3 \\
$\infty$ & 16 & $2 \pi^{1 / 2}$ & 201.1 \\
\hline
\end{tabular}

\section{Open channel cross-sections}

The conclusions reached above also hold for turbulent flow through a duct, in which the global flow resistance is more closely proportional to $p^{2} / A$, not Po $p^{2} / A$. This is relevant to understanding why there is a proportionality between width $(W)$ and maximum depth $(d)$ in rivers of all sizes (Leopold et al., 1964; Scheidegger, 1970). Because of the high Reynolds number and the roughness of the river bed, the skin friction coefficient $\mathrm{C}_{f}$ is essentially constant. The longitudinal shear stress along the river bottom is fixed ( $\tau=\frac{1}{2} C_{f} \rho V^{2}$ ) because $V=\dot{m} / \rho A$ and the mass flow rate $(\dot{m})$ and the river cross-sectional area $(A)$ are fixed. The total force per unit of channel length is $p \tau$, where $p$ is the wetted (bottom) perimeter of the cross-section. This means that the constructal law calls for cross-sectional shapes that have smaller $p$ values.

For example, if the cross-section is a rectangle of width $W$ and depth $d$, then $p=W+2 d$, and $A=W d$. The minimization of p subject to $A=$ constant yields $(W / d)_{\mathrm{opt}}=2$ and the $p_{\min } / A$ value shown in Table 2 . Other types of cross-sections can be optimized, and the resulting shape and performance are almost the same as for the rectangular case. The semicircular shape is the best, but it is not best by much. Once again, diversity of shapes on the podium of high performance is consistent with the constructal law. What is indeed random, because of local geological conditions (e.g. flat vs. curved river bottoms), coexists with pattern: the optimized aspect ratio and the minimized flow resistance $p_{\min } / A^{1 / 2}$.

In Table 2, the two most extreme cases are separated by only 12 percent in flow resistance. This high level of agreement with regard to performance is very important. It accounts for the significant scatter in the data on river bottom profiles, if global performance is what matters, not local shape. Again, this is in agreement with the new work on drainage basins (e.g. Sect. 5), where the computer-optimized (randomly generated) network looks like the many, never identical networks seen in the field. There is uncertainty in reproducing the many shapes that we see in Nature, but this is not important. There is very little uncertainty in anticipating global characteristics such as performance and geometric scaling laws (the ratio $W / d$ in this case).
Table 2. Optimized cross-sectional shapes of open channels (Bejan, 1997d).

\begin{tabular}{lll}
\hline Cross-section & $(W / d)_{\mathrm{opt}}$ & $p_{\min } / A^{1 / 2}$ \\
\hline Rectangle & 2 & 2.828 \\
Triangle & 2 & 2.828 \\
Parabola & 2.056 & 2.561 \\
Semicircle & 2 & 2.507 \\
\hline
\end{tabular}

Furthermore, in the optimal shape (half circle) the river banks extend vertically downward into the water and are likely to crumble under the influence of erosion (drag on particles) and gravity. This will decrease the slopes of the river bed near the free surface and, depending on the bed material, will increase the slenderness ratio $W / d$. The important point is that there remains plenty of room for the empiricism-based analyses of river bottoms proposed in geomorphology (Chorley et al., 1984), in fact, their territory remains intact. They complement constructal theory.

\section{Tree-shaped flows}

River basins and deltas, like the lungs and vascularized tissues of animal design, and like the tissues of social design and animal movement, are dendritic flow structures. The observed similarities between geophysical trees and biological trees have served as basis for empiricism: modeling in both fields, and descriptive algorithms in fractal geometry. In constructal theory, the thought process goes against the time arrow of empiricism (Fig. 2): first, the constructal law is invoked, and from it follows theoretically the deduced flow architecture. Only later is the theoretical configuration compared with natural phenomena, and the agreement between the two validates the constructal law.

In constructal theory tree-shaped flows are not models but solutions to fundamental access-maximization problems: volume-point, area-point and line-point. Important is the geometric notion that the "volume", the "area" and the "line" represent infinities of points. The theoretical discovery of trees in constructal theory stems from the decision to connect one point (source or sink) with the infinity of points (volume, area, line). It is the reality of the continuum (the infinity of points) that is routinely discarded by modelers who approximate the flow space as a finite number of discrete points, and then cover the space with "sticks", which (of course) cover the space incompletely (and, from this, fractal geometry).

Recognition of the continuum requires a study of the interstitial spaces between the tree links. The interstices can only be bathed by high-resistivity diffusion (an invisible, disorganized flow), while the tree links serve as conduits or lowresistivity organized flow (visible streams, ducts). Diffusion 
Biomimetics

Nature

Time

Engineering
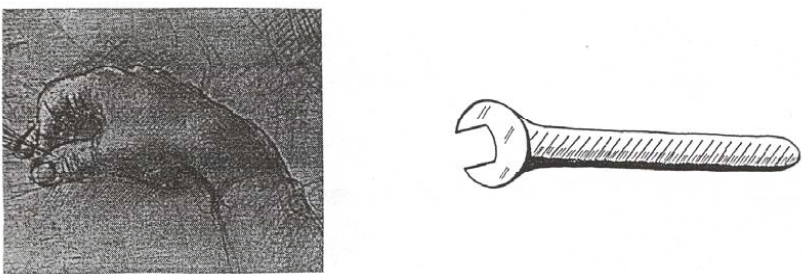

Constructal theory

Nature

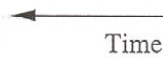

Principle
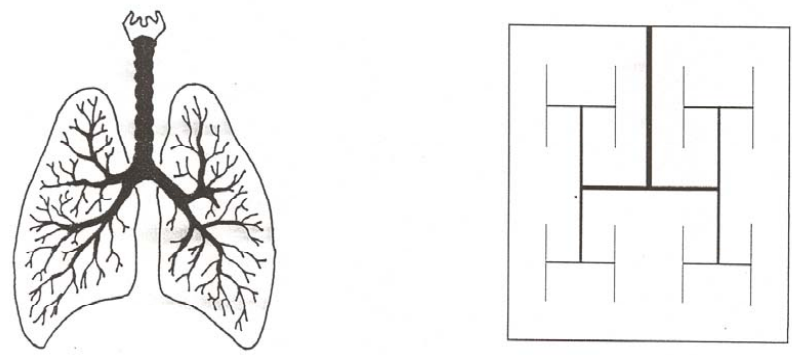

Fig. 2. Constructal theory proceeds in time against empiricism or copying from nature (Bejan, 2000).

is "disorganized" because the individuals that flow (fluid packets, molecules, etc.) flow individually, by interacting with their neighbors. Such individuals do not flow together. It is the latter, those flowing together that are "visible", as streams (currents) on the background of flow covered by diffusion. Diffusion does not have shape and structure. Stream flow does.

The two modes of flowing with imperfection (with flow resistance) - must be balanced so that together they contribute minimum imperfection to the global flow architecture. The flow architecture is the graphical expression of the balance between links and their interstices. The deduced architecture (tree, duct shape, spacing, etc.) is the optimal "distribution of imperfection". Those who model natural trees and then draw the branches as black lines (while not optimizing the layout of every black line on its allocated white patch) miss half of the drawing. The white is as important as the black.

The discovery of constructal tree-shaped flow architectures began with three approaches, two of which are reviewed here. The first was an analytical short cut Bejan (1996, 1997b, c) based on several simplifying assumptions: $90^{\circ}$ angles between stem and tributaries, a construction sequence in which smaller optimized constructs are retained, constantthickness branches, etc. At the same time, we considered the

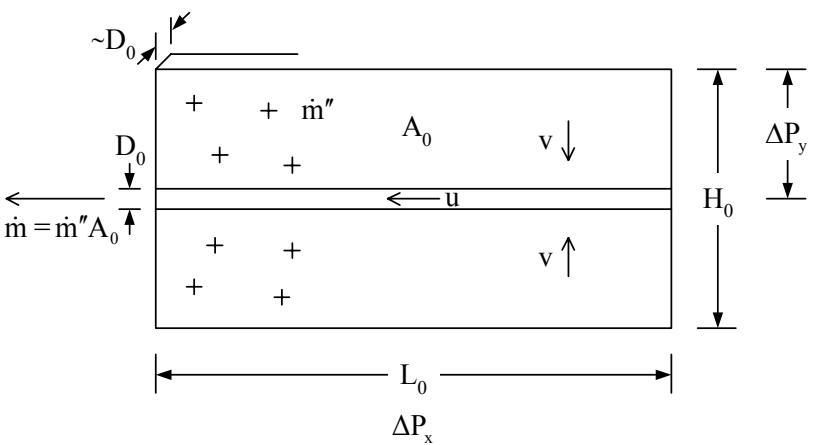

Fig. 3. Elemental area of a river basin viewed from above: seepage with high resistivity (Darcy flow) proceeds vertically, and channel flow with low resistivity proceeds horizontally. Rain falls uniformly over the rectangular area $A_{0}=H_{0} L_{0}$. The flow from the area to the point (sink) encounters minimum global resistance when the shape $H_{0} / L_{0}$ is optimized. The generation of geometry is the mechanism by which the area-point flow system assures its persistence in time, its survival.

same problem (Ledezma et al., 1997) numerically by abandoning most of the simplifying assumptions (e.g., the construction sequence) used in the first papers. The third approach was fully numerical (Bejan and Errera, 1998) in an area-point flow domain with random low-resistivity blocks embedded in a high-resistivity background, by using the language of Darcy flow (permeability, instead of thermal conductivity and resistivity). Along the way, we found better performance and "more natural looking" trees as we progressed in time; that is as we endowed the flow structure with more freedom to morph.

The first approach is illustrated in Fig. 3. The "elemental" area of a river basin $\left(A_{0}=H_{0} L_{0}\right)$ is the area allocated to the smallest rivulet (length $L_{0}$, width $D_{0}$, depth scale $Z$, where, as shown in Sect. $4, Z$ scales with $D_{0}$. Rain falls uniformly on $A_{0}$ with the mass flow rate $\dot{m}^{\prime \prime}\left[\mathrm{kg} \mathrm{s}^{-1} \mathrm{~m}^{-2}\right]$. Constructal theory predicts an optimal allocation of area to each channel: there is an optimal elemental shape $H_{0} / L_{0}$ such that the total flow rate $\left(\dot{m}^{\prime \prime} A_{0}\right)$ collected on $A_{0}$ escapes with least global flow resistance from $A_{0}$ through one port on its periphery. For example, if the water seepage through the wet banks (perpendicular to the rivulet) is in the Darcy flow regime, then the pressure (or elevation) difference that derives the seepage velocity $\mathrm{v}$ is of order $\Delta P_{y} \sim v \mu H_{0} / K$, where $K$ is the permeability of the porous medium. If the rivulet flow is in the Poiseuille regime, then the pressure (or elevation) drop along the $L_{0}$ rivulet is of order $\Delta P_{x} \sim u \mu L_{0} / D_{0}^{2}$. Here $u$ is the mean fluid velocity along $L_{0}$. These equations can be combined to conclude that the overall pressure difference that drives the area-point flow is

$\Delta P_{x}+\Delta P_{y} \sim \dot{m} v\left(\frac{L_{0}}{D_{0}^{2}}+\frac{H_{0}}{K L_{0} D_{0}}\right)$ 


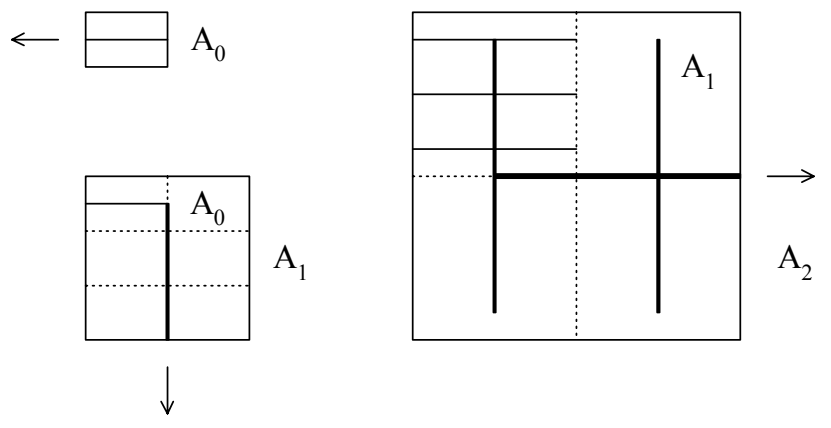

Fig. 4. Constructal sequence of assembly and optimization, from the optimized elemental area $\left(A_{0}\right.$, Fig. 3$)$ to progressively larger area-point flows.

The derivation of Eq. (2) is detailed in Bejan (1997d, 2000). This expression can be minimized with respect to the shape of the area element, and the result is

$$
\left(\frac{L_{0}}{H_{0}}\right)_{\mathrm{opt}} \sim\left(\phi_{0} \frac{A_{0}}{K}\right)^{1 / 3}
$$

where $\phi_{0}$ is the area fraction occupied by the rivulet on the flow map, $\phi_{0}=D_{0} L_{0} / H_{0} L_{0} \ll 1$. When the area element has optimal shape, $\Delta P_{y}$ is of the same order as $\Delta P_{x}$. This is a frequent occurrence in the maximization of area-point flow access: the optimal partitioning of the driving force between the two flow mechanisms is synonymous with the optimization of area geometry (Lewins, 2003).

The optimized area element becomes a building block with which larger rain plains can be covered. The elements are assembled and connected into progressively larger area constructs, in a sequence of assembly with optimization at every step. During this sequence, the river channels form a tree architecture in which every geometric detail is deduced, not assumed. The construction is illustrated in Fig. 4, and in the current literature (Neagu and Bejan, 1999; Lundell et al., 2004; Kockman et al., 2005). For river basins with constant$C_{f}$ turbulent flow, the constructal sequence shows that the best rule of assembly is not doubling but quadrupling (Bejan, 2006) (e.g. $A_{2}=4 A_{1}$ in Fig. 4) and that river basins deduced in this manner exhibit all the Hortonian scaling relationships observed in natural river basins (Bejan, 2006, Sect. 13.5).

Another approach to deducing tree-shaped drainage basins from the constructal law is presented in Bejan and Errera (1998) and Fig. 5. The two flow regimes are seepage (Darcy flow) through regions of low permeability $(K)$, and seepage through high-permeability regions $\left(K_{p}\right)$ created by grains that have been removed (eroded). The surface area $A=H L$ and its shape $H / L$ are fixed. The area is coated with a homogeneous porous layer of permeability $K$. The small thickness of the $K$ layer, i.e., the dimension perpendicular to the plane $H \times L$, is $W$, where $W \ll(H, L)$.

An incompressible Newtonian fluid is pumped through one of the $A$ faces of the $A \times W$ parallelepiped, such that the
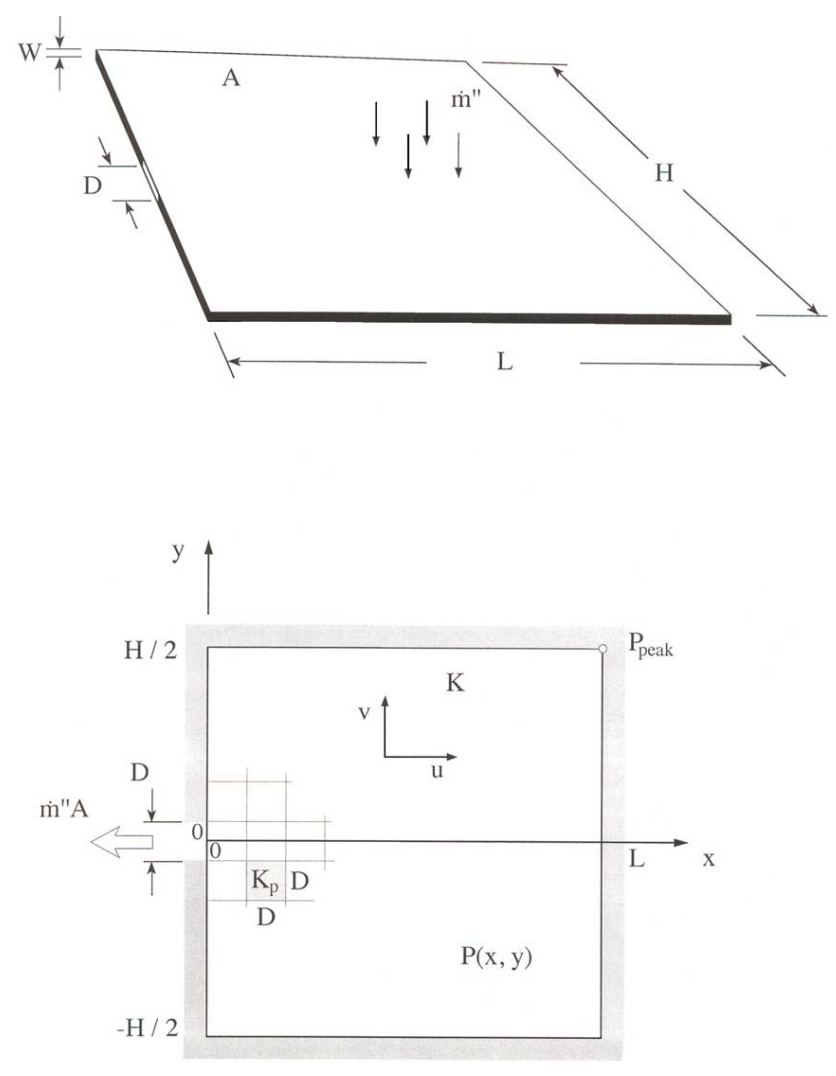

Fig. 5. Area-point flow in a porous medium with Darcy flow and grains that can be dislodged and swept downstream (Bejan and Errera, 1998).

mass flow rate per unit area is uniform, $\dot{m} "\left[\mathrm{~kg} / \mathrm{m}^{2} \mathrm{~s}\right]$. The other $A$ face and most of the perimeter of the $H \times L$ rectangle are impermeable. The collected stream $(\dot{m} " A)$ escapes through a small port of size $D \times W$ placed over the origin of the $(x, y)$ system. The fluid is driven to this port by the pressure field $P(x, y)$ that develops over $A$. The pressure field accounts for the effect of slope and gravity in a real river drainage basin, and the uniform flow rate $\dot{m}$ " accounts for the rainfall.

The global resistance to this area-to-point flow is the ratio between the maximal pressure difference $\left(P_{\text {peak }}\right)$ and the total flow rate $(\dot{m} " A)$. The location of the point of maximal pressure is not the issue, although in Fig. 5 its position is clear. It is important to calculate $P_{\text {peak }}$ and to reduce it at every possible turn (in time) by making appropriate changes in the internal structure of the $A \times W$ system. Determinism results from invoking a single principle and using it consistently.

Changes are possible because finite-size portions (blocks, grains) of the system can be dislodged and ejected through the outlet. The removable blocks are of the same size and shape (square, $D \times D \times W$ ). The critical force (in the plane of $A$ ) that is needed to dislodge one block is $\tau D^{2}$, where 

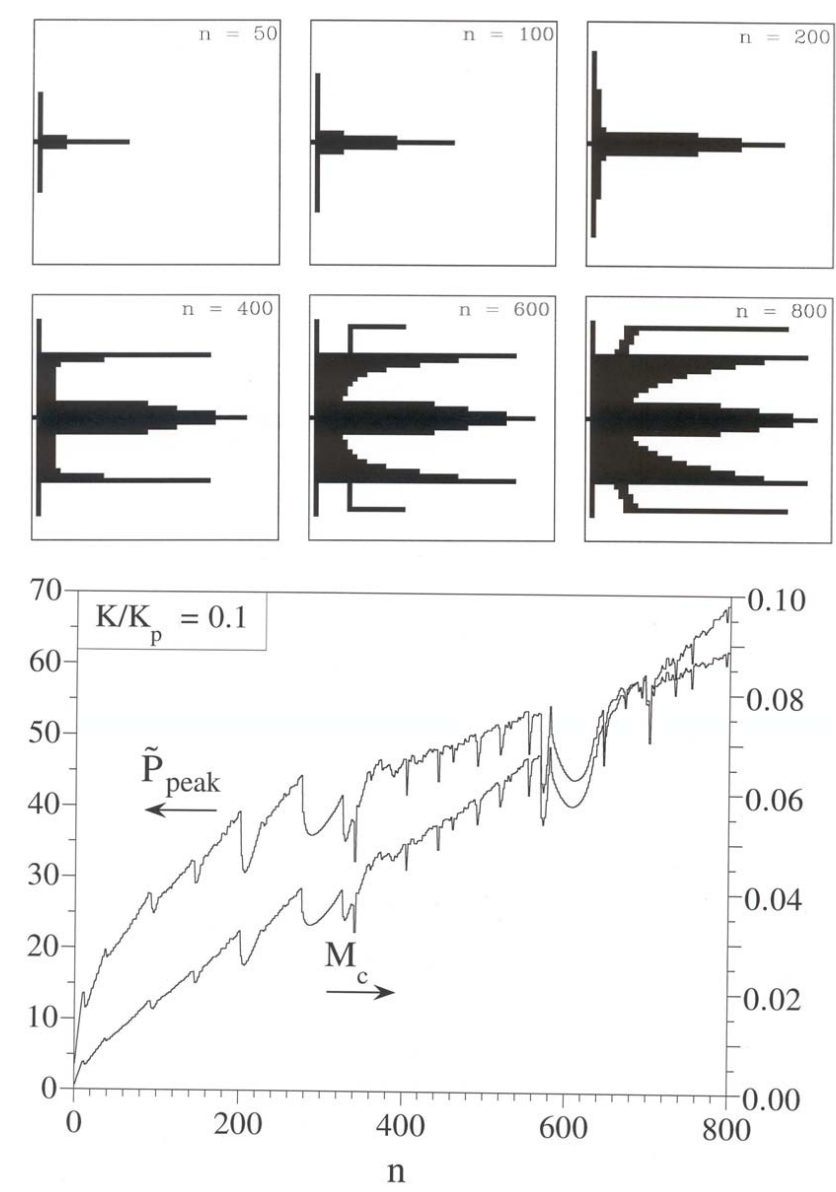

Fig. 6. The evolution (persistence, survival) of the tree structure when $K / K_{p}=0.1$ (Bejan and Errera, 1998).

$\tau$ is the yield shear stress averaged over the base area $D^{2}$. The yield stress and the length scale D are assumed known. They provide an erosion criterion and a useful estimate for the order of magnitude of the pressure difference that can be sustained by the block. At the moment when one block is dislodged, the critical force $\tau D^{2}$ is balanced by the net force induced by the local pressure difference across the block $\Delta P$, namely $\triangle P D W$. The balance $\tau D^{2} \sim \triangle P D W$ suggests the pressure-difference scale $\Delta P \sim \tau D / W$, which along with $D$ can be used for the purpose of nondimensionalizing the problem formulation. For example, the dimensionless pressure difference is $\tilde{P}=P /(\tau D / W)$, and the intensity of the rainfall is described by the dimensionless number $M=\dot{m}^{\prime \prime} v D /(\tau K)$.

A simple way to model erosion is to assume that the space vacated by the block is also a porous medium with Darcy flow except that the new permeability $\left(K_{p}\right)$ of this medium is sensibly greater, $K_{p}>K$. This assumption is correct when the flow is slow enough (and $W$ is small enough) so that the flow regime in the vacated space is Hagen-Poiseuille between parallel plates. The equivalent $K_{p}$ value for such a flow is $W^{2} / 12$ (cf. Bejan, 2004).
The pressure $\tilde{P}$ and the block-averaged pressure gradient increase in proportion with the imposed mass flow rate $(M)$. The mass flow rate is "imposed" because in this scenario $\dot{m}^{\text {" }}$ plays the role of the artificial (imposed) rainfall in laboratory simulations of the evolution of river basins (e.g., Bejan, 1997d). When $M$ exceeds a critical value $M_{c}$, the first block is dislodged. The physics principle that we invoke is this: the resistance to fluid flow is decreased through geometric changes in the internal architecture of the system. To generate higher pressure gradients that may lead to the removal of a second block, we must increase the flow-rate parameter $M$ above the first $M_{c}$, by a small amount. The removable block is one of the blocks that borders the newly created $K_{p}$ domain. The peak pressure rises as $M$ increases, and then drops partially as the second block is removed. This process can be repeated in steps marked by the removal of each additional block. In each step, we restart the process by increasing $M$ from zero to the new critical value $M_{c}$. During this sequence the peak pressure decreases, and the overall area-to-point flow resistance $\left(\tilde{P}_{\text {peak }} / M_{c}\right)$ decreases monotonically.

The key result is that the removal of certain blocks of $K$ material and their replacement with $K_{p}$ material generate macroscopic internal structure. The mechanism and the resulting structure are deterministic: every time we repeat this process we obtain exactly the same sequence of images.

For illustration, consider the case $K / K_{p}=0.1$, shown in Fig. 6. The number $n$ on the abscissa represents the number of blocks that have been removed. The domain $A$ is square and contains a total of 2601 building blocks of base size $D \times D$; in other words, $H=L=51 D$. Figure 6 also shows the evolution of the critical flow rate and peak pressure. The curves appear ragged because of an interesting feature of the erosion model: every time that a new block is removed, the pressure gradients redistribute themselves and blocks that used to be "safe" are now ready to be dislodged even without an increase in $M$. The fact that the plotted $M_{c}$ values drop from time to time is due to restarting the search for $M_{c}$ from $M=0$ at each step $\mathrm{n}$.

The shape of the high-permeability domain $K_{p}$ that expands into the low-permeability material $K$ is that of a tree. New branches grow in order to channel the flow collected by the low-permeability $K$ portions. The growth of the first branches is stunted by the fixed boundaries (size, shape) of the $A$ domain. The older branches become thicker; however, their early shape (slenderness) is similar to the shape of the new branches.

The slenderness of the $K_{p}$ channels and the interstitial $K$ regions is dictated by the $K / K_{p}$ ratio, that is, by the degree of dissimilarity between the two flow paths. Highly dissimilar flow regimes $\left(K / K_{p} \ll 1\right)$ lead to slender channels (and slender $K$ interstices) when the overall area-to-point resistance is minimized. On the other hand, when $K / K_{p}$ is close to 1 , channels (fingers) do not form: the eroded region grows as a half disc (Bejan and Errera, 1998). 
Figure 6 stresses the observation that the availability of two dissimilar flow regimes $\left(K_{p} \neq K\right)$ is a necessary precondition for the formation of deterministic structures through flow-resistance minimization. The "glove" is the highresistance regime $(K)$, and the "hand" is the low-resistance regime $\left(K_{p}\right)$ : the two regimes work "hand in glove" toward minimizing the overall resistance.

The raggedness of the $\tilde{P}_{\text {peak }}(n)$ curves disappears when the flow-rate parameter $M$ is increased monotonically from one step to the next (e.g., Fig. 7). Each step begins with the removal of the first block that can be dislodged by the flow rate $M$. Following the removal of the first block, the $M$ value is held fixed, the pressure field is recalculated and the block removal criterion is applied again to the blocks that border the newly shaped $K_{p}$ domain. To start the next step, the $M$ value is increased by a small amount $\Delta M$. The $M(n)$ curves shown in Fig. 7 are stepped because of the assumed size of $\Delta M$ and the finite number $(\Delta n)$ of blocks that are removed during each step. Although the monotonic $M(n)$ curves obtained in this manner are not the same as the critical flow-rate curves $M_{c}(n)$ plotted in Fig. 6, they too are deterministic.

Figure 7 corresponds to a composite porous material with $K / K_{p}=0.1$, which is the same material from which the river basin of Fig. 6 was constructed. Compare the shapes of the high-conductivity domains shown in these figures. The handin-glove structure is visible in all three figures; however, the finer details of the $K_{p}$ domain depend on how the flow rate $M$ is varied in time. The main difference between the patterns of Fig. 6 and those of Fig. 7 is visible relatively early in the erosion process: Diagonal fingers form when the flow rate is increased monotonically. In conclusion, the details of the internal structure of the system depend on the external "forcing" that drives it, in our case the function $M(n)$. The structure is deterministic, because it is known when the function $M(n)$ is known.

Major differences exist between natural river drainage structures and the deterministic structures illustrated in Figs. 6 and 7. One obvious difference is the lack of symmetry in natural river trees. How do we reconcile the lack of symmetry and unpredictability of the finer details of a natural pattern with the deterministic mechanism that led us to the discovery of tree networks of Figs. 6 and 7? The answer is that the developing structure depends on two entirely different concepts: the generating mechanism, which is deterministic, and the properties of the natural flow medium, which are not known accurately and at every point.

In developing Fig. 8, we assumed that the resistance that characterizes each removable block is distributed randomly over the basin area. This characteristic of river beds is well known in the field of river morphology (Leopold et al., 1964). For the erosion process we chose the system $\left(K / K_{p}=0.1\right)$ and the $M(n)$ function of Fig. 7, in which $M$ increased monotonically in steps of 0.001 . The evolution of the drainage system is shown in Fig. 8. The emerging tree network is considerably less regular than in Fig. 7, and reminds us more
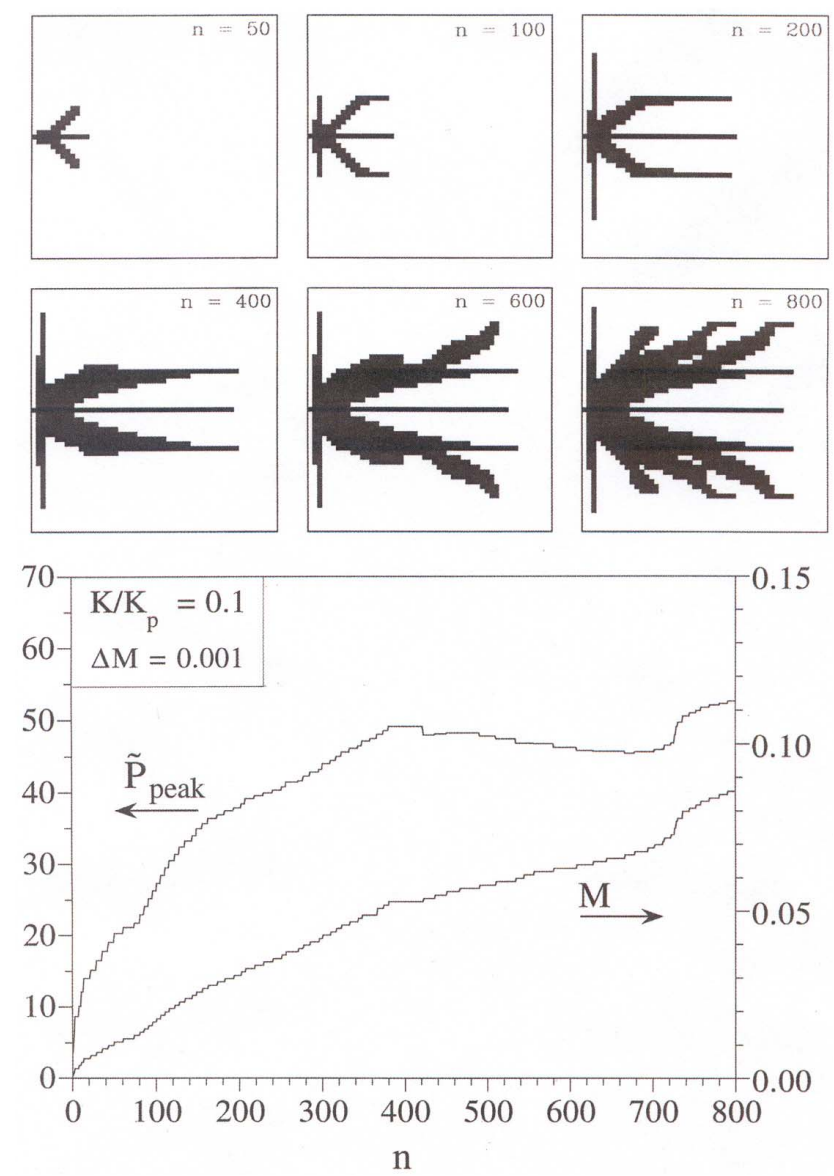

Fig. 7. The evolution (persistence, survival) of the tree structure when $K / K_{p}=0.1$ and the flow rate $M$ is increased in steps $\Delta M=10^{-3}$ (Bejan and Errera, 1998).

of natural river basins. The unpredictability of this pattern, however, is due to the unknown spatial distribution of system properties, not to the configuration-generating principle (the constructal law), which is known.

The natural phenomenon of river basin generation is similar to the time sequences shown in Figs. 6-8. See for example, the sequence of drawings of the development of an artificial river basin over a $15.2 \mathrm{~m} \times 9.1 \mathrm{~m}$ rainfall erosion area (Parker, 1977; reproduced as Fig. 13.19 in Bejan, 2006). At the start, there is no drawing. In time, the tree drawing flows better and better, and in each time frame the drawing is treeshaped. There are similarities and differences between these images and numerical simulations that appear in the hydrology literature. For example, Rodriguez-Iturbe et al. (1992) modeled the river basin by postulating the existence of a large number of channels on a rectangular domain (one channel for each little square element of the domain) and then moving the channels randomly on the computer such that the global flow resistance of basin is minimized (recall EGM). After enough random modifications of the assembly of line channels, the 

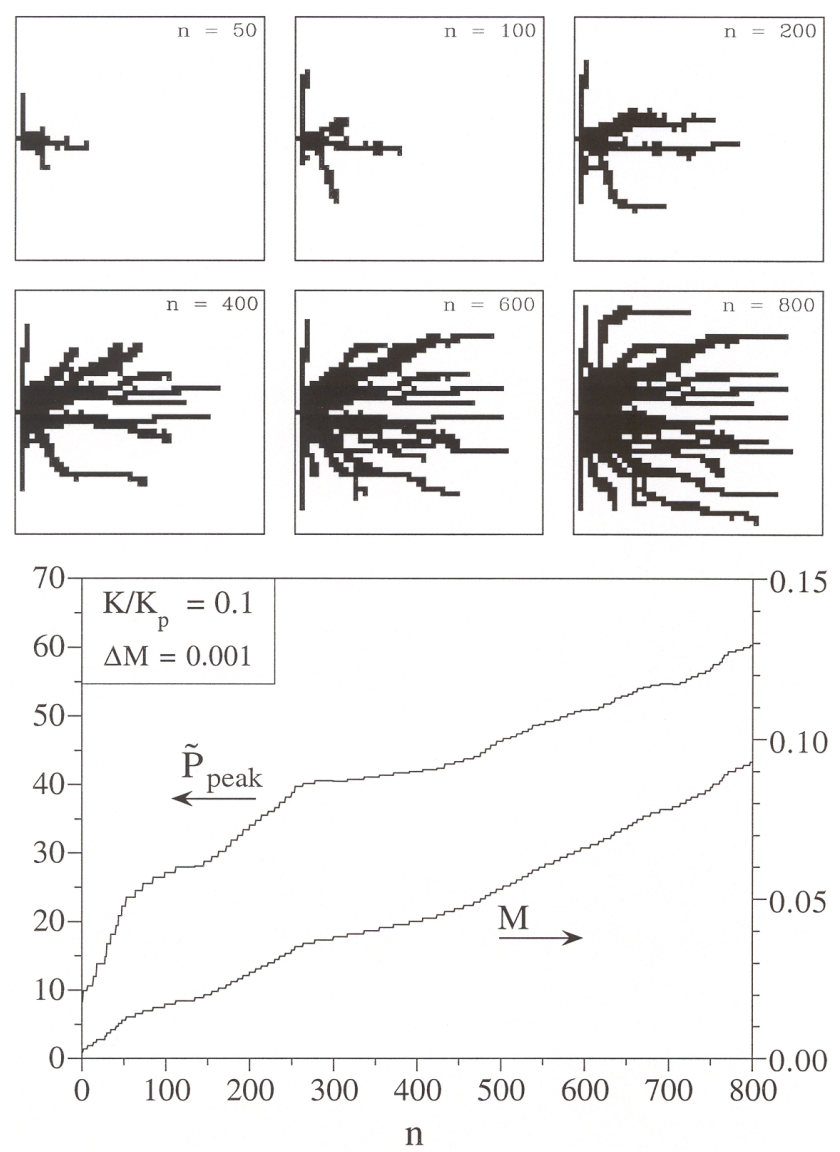

Fig. 8. The evolution (persistence, survival) of the tree structure in a random-resistance erodable domain, when $\mathrm{K} / \mathrm{K}_{p}=0.1$ and $\mathrm{M}$ increases in steps of 0.001 (Bejan and Errera, 1998).

ultimate pattern becomes dendritic, irregular and similar to what we see in nature and the frames of Figs. 6-8. If the process is repeated, the sequence of modifications is different, the ultimate pattern is different, but it is once again dendritic and irregular.

In such ad-hoc invocation of EGM, the focus is on the end objective and pattern. In constructal theory, the story is the time direction of the changes in flow pattern, in which the sequence of drawings is unique, like the sequence of natural drawings (Parker, 1977). Another important difference is that the flow along the smallest channel is as important (i.e. in balance with) the seepage perpendicular to the channel (see again Fig. 3). Channels and hill slopes are allocated optimally to each other. This is unlike in the numerical simulations of Rodriguez-Iturbe et al. (1992), where the smallest area elements and channels are of one size and postulated, and where the global flow resistance accounts only for the cumulative resistance of the channels.

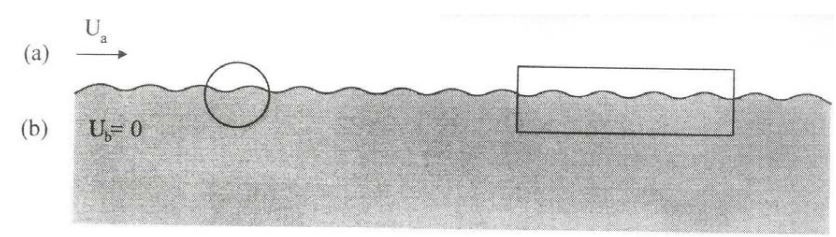

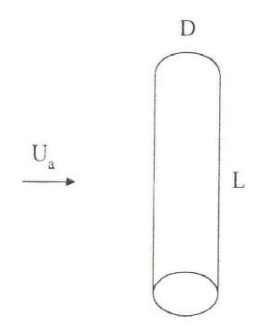

(1)

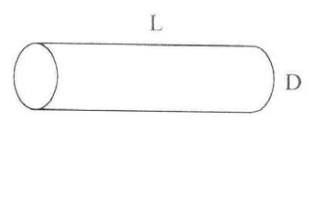

(2)
Fig. 9. Floating object at the interface between two fluid masses with relative motion (Bejan, 2000).

\section{Turbulent flow structure}

A turbulent flow has "structure" because it is a combination of two flow mechanisms: viscous diffusion and streams (eddies). Both mechanisms serve as paths for the flow of momentum. According to the constructal law, the flow structure called "turbulence" is the architecture that provides the most direct path for the flow of momentum from the fast regions of the flow field to the slow regions (Bejan, 1997d, 2000).

This tendency of optimizing the flow configuration so that momentum flows the easiest is illustrated in Fig. 9. An object (iceberg, tree $\log$ ) floats on the surface of the ocean. The atmosphere (a) moves with the wind speed $U_{a}$, while the ocean water $(b)$ is stationary. If $(a+b)$ form an isolated system initially far from equilibrium, the constructal law calls for the generation of flow configuration that brings (a) and (b) to equilibrium the fastest. The floating object is the "key" mechanism by which (a) transfers momentum to (b). The extreme configurations of this mechanism are (1) and (2). The forces with which (a) pulls (b) are

$$
F_{1} \sim L_{D} C_{D} \frac{1}{2} \rho_{a} U_{a}^{2} \quad F_{2} \sim D^{2} C_{D} \frac{1}{2} \rho_{a} U_{a}^{2}
$$

where the drag coefficient $C_{D}$ is a factor of order 1 . The constructal configuration is (1), because $F_{1}>F_{2}$ when $L>\mathrm{D}$. This is confirmed by all objects that drift on the ocean: icebergs, debris, abandoned ships, etc.

The turbulent eddy is the equivalent key mechanism when momentum access is maximized between two regions of the same flowing fluid. Instead of the air and water shear flow of Fig. 9, in Fig. 10 we consider the shear flow between fast and slow regions of the same fluid (a). Configuration (1) is the laminar shear flow (viscous diffusion), where the shear stress at the (a)-(a) interface is $\tau_{1} \sim \mu U_{\infty} / D$. Configuration 
(a)

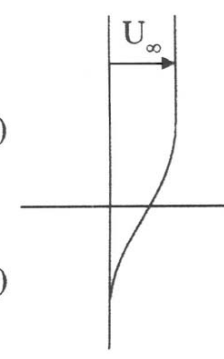

(1)

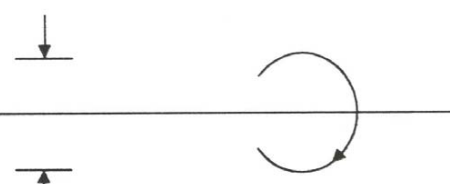

(2)

streams (eddy) viscous diffusion

Fig. 10. The two momentum-transfer mechanisms that compete at the interface between two flow regions of the same fluid (Bejan, 2000).

(2) is the eddy flow: the wrinkling, rolling and thickening of the shear. The rolls have the peripheral speed $U_{\infty}$. The roll is a counterflow that transfers horizontal momentum in the downward direction [from (a) to (a)] at the rate $\left(\rho D U_{\infty}\right) U_{\infty}$. The rate of momentum transfer per unit of interface area in two dimensions is $\tau_{2} \sim \rho D U_{\infty} U_{\infty} / D$.

Rolls (eddies) are a necessary constructal feature of the prevailing flow architecture when $\tau_{2}>\tau_{1}$, which yields $U_{\infty} D / \nu>1$. More precise evaluations of $\tau_{1}$ and $\tau_{2}$, substituted into $\tau_{2}>\tau_{1}$, yield the local Reynolds number criterion for the formation of the first eddies:

$R e_{l}=\frac{U_{\infty} D}{v}>O\left(10^{2}\right)$

This prediction is supported convincingly by the laminarturbulent transition criteria reviewed in Table 3. The traditional criteria are stated in terms of critical numbers that range from 30 to $4 \times 10^{12}$. All the $\operatorname{Re}_{l}$ equivalents of these classical observations agree with $\operatorname{Re}_{l} \sim 10^{2}$ at transition.

The main theoretical development is that the constructal law accounts for the occurrence of eddies - eddies in the eye of the mind where, before the invocation of the law, eddies were alien (not known) as a happening, drawing and concept. Each eddy is an expression of the optimal balance between two momentum transport mechanisms (cf. $\tau_{1} \sim \tau_{2}$ ), in the same way that every rivulet is in balance with the seepage across the area allocated to the rivulet (cf. Fig. 3). For the first time in the physics of fluid flow, the eddy structure is deduced, not assumed (the eddy is not an assumed and over grown "disturbance").

The support for the theoretical view of turbulence as a constructal configuration-generation phenomenon is massive. Table 3 is one example of how an entire chapter of fluid mechanics is replaced by a single theoretical formula, Eq. (5). Another example is Fig. 11, which shows a large number of measurements of the laminar length $\left(L_{t r}\right)$ in the best known

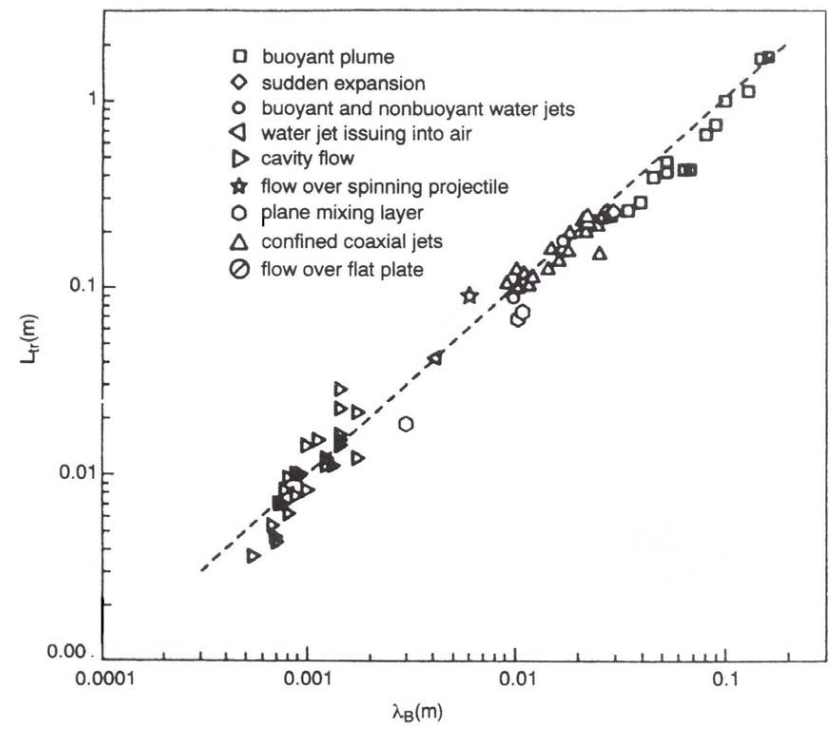

Fig. 11. The universal proportionality between the length of the laminar section and the buckling wavelength in a large number of flows (Bejan, 2004).

flow configurations, versus the buckling wavelength $\left(\lambda_{B}\right)$ in the transition zone. All the data are correlated by the line

$\frac{L_{t r}}{\lambda_{B}} \sim 10$

It was shown in Bejan (2004) that this proportionality can be predicted by invoking Eq. (5).

Other features of turbulent structure that have been deduced from the constructal law are the wedge shape (selfsimilar region) of turbulent shear layers, jets and plumes, the Strouhal number associated with vortex shedding, Bénard convection in fluids and fluid-saturated porous media heated from below, etc. These developments are reviewed in Bejan (1997d, 2000). This approach has been taken to cover all scales, to predict purely theoretically the main features of global atmospheric and oceanic circulation and climate (Bejan and Reis, 2005; Reis and Bejan, 2006), the morphology of liquid droplets that impact a wall (splat vs. splash, cf. Bejan and Gobin, 2006), and the dendritic clustering of dust particles (Reis et al., 2006). It was also used to predict dendritic solidification (snowflakes), dendritic evaporation (vegetation) and the coalescence of solid parcels suspended in flow Bejan (1997d, 2000). Many more classes of natural flow architectures that obey the constructal law have been described in biology, from the necessity of intermittent breathing and heartbeating, to the scaling laws of all animal locomotion (running, flying, swimming) (Bejan, 2000, 2006; Bejan and Marden, 2006). 
Table 3. Traditional critical numbers for transitions in several key flows and the corresponding local Reynolds number (Bejan, 2000).

\begin{tabular}{|c|c|c|}
\hline Flow & $\begin{array}{l}\text { Traditional } \\
\text { Critical Number }\end{array}$ & $\begin{array}{l}\text { Local } \\
\text { Reynolds } \\
\text { Number }\end{array}$ \\
\hline Boundary-layer flow over flat plate & $\operatorname{Re}_{x} \sim 2 \times 10^{4}-10^{6}$ & $\operatorname{Re}_{1} \sim 94-660$ \\
\hline $\begin{array}{l}\text { Natural convection boundary layer along vertical wall with uniform } \\
\text { temperature }(\operatorname{Pr} \sim 1)\end{array}$ & $\mathrm{Ra}_{y} \sim 10^{9}$ & $\operatorname{Re}_{1} \sim 178$ \\
\hline $\begin{array}{l}\text { Natural convection boundary layer along vertical wall with constant } \\
\text { heat flux }(\operatorname{Pr} \sim 1)\end{array}$ & $\mathrm{Ra}_{* y} \sim 4 \times 10^{12}$ & $\operatorname{Re}_{1} \sim 330$ \\
\hline Round jet & $\operatorname{Re}_{\text {nozzle }} \sim 30$ & $\operatorname{Re}_{1} \geq 30$ \\
\hline Wake behind long cylinder in cross flow & $\operatorname{Re} \sim 40$ & $\operatorname{Re}_{1} \geq 40$ \\
\hline Pipe flow & $\operatorname{Re} \sim 2000$ & $\operatorname{Re}_{1} \sim 500$ \\
\hline Film condensation on a vertical wall & $\operatorname{Re} \sim 450$ & $\operatorname{Re}_{1} \sim 450$ \\
\hline
\end{tabular}

\section{Mathematical formulation of the constructal law}

Professor K. Roth, the editor in chief of this journal, made the important observation that laws of physics are invariably expressed in mathematical statements, i.e. that the constructal law cited in Sect. 1 is deficient in this respect. I agree, and in this section I show how we have formulated the constructal law mathematically in analytical geometry (Bejan and Lorente, 2003, 2004, 2005). It is worth noting however that the history of the evolution of science (e.g. Bejan, 2006, Sect. 13.9) shows that it takes time before a new idea is expressed in crisp mathematical terms. Because the subject here is the thermodynamics of nonequilibrium (flow) systems, recall S. Carnot's mental viewing of heat flowing from high to low temperature through a steam engine, "like river water through a turbine". S. Carnot said in prose the essence of thermodynamics. His vision was put into mathematical terms threee decades later by R. Clausius, who invented for this purpose the concept and property called entropy. But even then, after the math, when the new laws needed help to be explained to the public, Clausius had to resort to bombastic prose to demystify the math (entropy) that he invented (see his famous line: "Die Energie der Welt ist constant. Die Entropie der Welt strebt einem Maximum zu".).

Just like Clausius, in order to mathematize the constructal law we had to define new properties for a thermodynamic system that has configuration. These properties distinguished it from a static (equilibrium, nothing flows) system, which does not have configuration. The properties of a flow system are:

(1) global external size, e.g., the length scale of the body bathed by the tree flow $L$;
(2) global internal size, e.g., the total volume of the ducts $\checkmark$;

(3) at least one global measure of performance, e.g., the global flow resistance of the tree $R$;

(4) configuration, drawing, architecture; and

(5) freedom to morph, i.e., freedom to change the configuration.

The global external and internal sizes $(L, V)$ mean that a flow system has at least two length scales $L$ and $V^{1 / 3}$. These form a dimensionless ratio - the svelteness $\mathrm{Sv}$ - which is a new global property of the flow configuration (Lorente and Bejan, 2005).

$\mathrm{Sv}=\frac{\text { external length scale }}{\text { internal length scale }}=\frac{L}{V^{1 / 3}}$.

(a) Survival by increasing flow performance

Figure 12 was drawn for constant $L$ : the global size is the same for all the flow architectures that are represented by this figure. The constructal law (Sect. 1) is the statement that summarizes the common observation that flow structures that survive are those that morph (evolve) in one direction in time: toward configurations that make it easier for currents to flow. This statement refers strictly to structural changes under finite-size constraints. If the flow structures are free to change (free to approach the base plane in Fig. 12), in time they will move at constant- $L$ and constant- $V$ in the direction of progressively smaller $R$. If the initial configuration 
is represented by point 1 , then a later configuration is represented by point 2 . The constructal law requires

$R_{2} \leq R_{1}$ (constant $\left.L, V\right)$

If freedom to morph persists, then the flow structure will continue toward smaller $R$ values. Any such change is characterized by

$d R \leq 0($ constant $L, V)$

The end of this migration is the "equilibrium flow structure", where the geometry of the flow enjoys total freedom. Equilibrium is characterized by minimal $R$ at constant $L$ and $V$. In the vicinity of the equilibrium flow structure we have

$d R=0 \quad$ and $\quad d^{2} R>0($ constant $L, V)$

The $R(V)$ curve shown in the bottom plane of Fig. 12 is the edge of the cloud of possible flow architectures with the same global size $L$. The curve has negative slope because of the physics of flow: the resistance decreases when the flow channels open up:

$$
\left(\frac{\partial R}{\partial V}\right)_{L}<0
$$

In summary, the evolution of configurations in the constant$V$ cut (also at constant $L$, Fig. 12) represents survival through increasing performance - survival of the fittest. This is the physics principle that finally underpins the Darwinian argument, the physics law that rules not only the animate flow systems but also the natural inanimate flow systems and all the man and machine species. The constructal law defines the meaning of "the survivor", or of the equivalent concept of "the more fit". The constructal-law idea that freedom to morph is good for performance (Fig. 12) also accounts for the Darwinian argument that the survivor is the one most capable to adapt.

In the bottom plane of Fig. 12, the locus of equilibrium structures is a curve with negative slope. The time evolution of nonequilibrium flow structures toward the bottom edge of the surface (the equilibrium structures) is the action of the constructal law.

\section{(b) Survival by increasing svelteness}

The same time arrow can be described alternatively with reference to the constant- $R$ cut through the threedimensional space of Fig. 12. Flow architectures with the same global performance $(R)$ and global size $(L)$ evolve toward compactness and svelteness - smaller volumes dedicated to internal ducts, i.e., larger volumes reserved for the working "tissue" (the interstices). Paraphrasing the original statement of the constructal law, we may describe the evolution at constants $L$ and $R$ as follows:

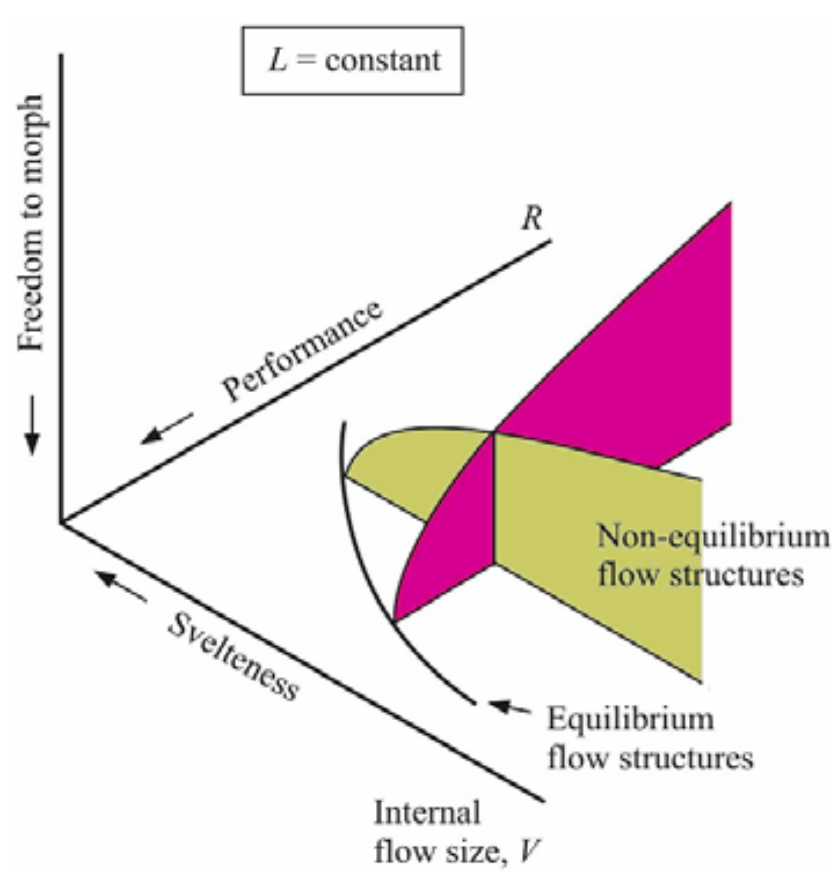

Fig. 12. Performance vs. freedom to change configuration, at fixed global external size (Bejan and Lorente, 2003, 2004).

For a system with fixed global size and global performance to persist in time (to live), it must evolve in such a way that its flow structure occupies a smaller fraction of the available space.

This is survival based on the maximization of the use of the available space. Survival by increasing svelteness (compactness) is equivalent to survival by increasing performance: both statements are the constructal law.

\section{(c) Survival by increasing flow territory}

A third equivalent statement of the constructal law becomes evident if we recast the constant- $L$ design world of Fig. 12 in the constant- $V$ design space of Fig. 13. In this new figure, the constant- $L$ cut is the same performance versus freedom diagram as in Fig. 12, and the constructal law means survival by increasing performance. The contribution of Fig. 13 is the shape and orientation of the hypersurface of nonequilibrium flow structures: the slope of the curve in the bottom plane $(\partial R / \partial L)_{V}$ is positive because of physics (fluid mechanics), i.e., because the flow resistance increases when the distance traveled by the stream increases.

The world of possible designs can be viewed in the constant- $R$ cut made in Fig. 13, to see that flow structures of a certain performance level $(R)$ and internal flow volume $(V)$ morph into new flow structures that cover progressively larger territories. Again, flow configurations evolve toward greater svelteness Sv. The constructal law statement becomes: 


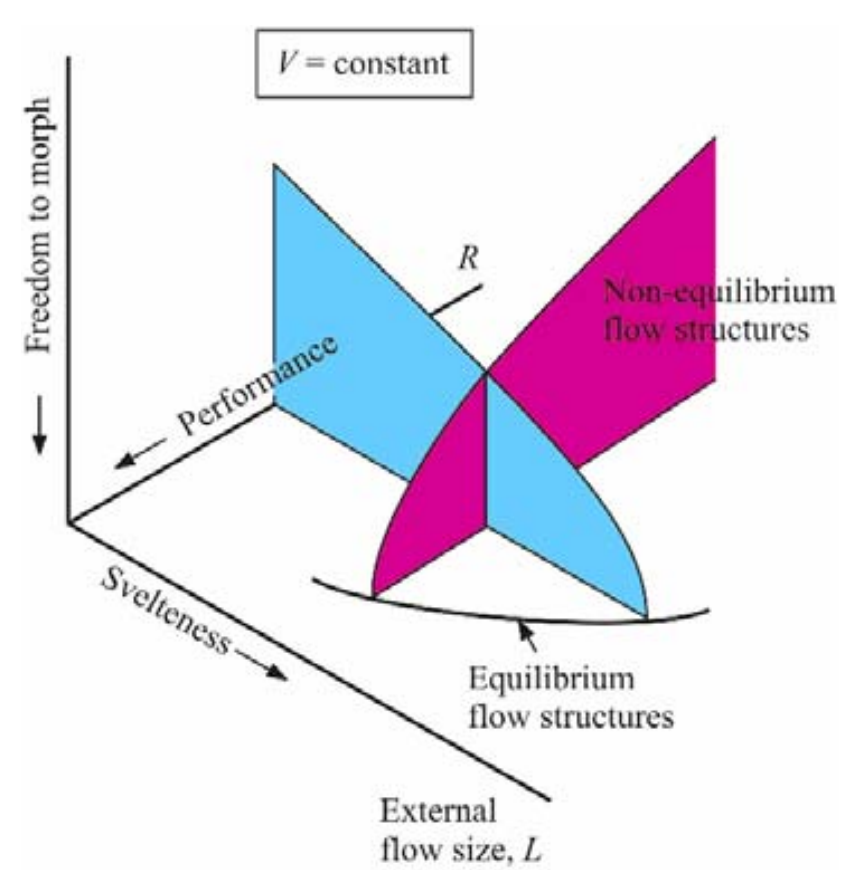

Fig. 13. Performance vs. freedom to change configuration, at fixed global internal size (Bejan and Lorente, 2003, 2004).

In order for a flow system with fixed global resistance $(R)$ and internal size $(V)$ to persist in time, the flow architecture must evolve in such a way that it covers a progressively larger territory.

There is a limit to the spreading of a flow structure, and it is set by global properties such as performance (technology) and internal flow volumes $R$ and $V$. River deltas in the desert, animal species on the plain, and the Roman empire spread in time to their constructal limits. Such is the constructal law of survival by spreading, by increasing territory for flow and movement.

\section{A place for theory}

In summary, it is possible to rationalize and predict the occurrence of flow configuration in nature on the basis of a principle of physics: the constructal law. The importance of this development in fields such as hydrology is greater because it has the potential of changing the way in which research is pursued.

Hydrology research is proving every day that science has hit a wall. Principles such as Newton's second law of motion (the Navier-Stokes equations) are not enough. Because of progressively more powerful computational and information gathering tools, models are becoming more complex, with more empirical features to be fitted to measurements. They provide better description, not explanation. They do not provide a mental viewing of how things should be. They are not theory.
What holds for contemporary hydrology also holds for other extremely active fields such as turbulence research and biology. Needed are principles with the same universal reach as that of Newton's second law of motion and the first and second laws of thermodynamics. Needed are new laws of physics. A prerequisite or success on this path is a new attitude: physics is not and never will be complete.

Physics is our knowledge of how nature (everything) works. Our knowledge is condensed in simple statements (thoughts, connections), which evolve in time by being replaced by simpler statements. We "know more" because of this evolution in time. Our finite-size brains keep up with the steady inflow of new information through a process of simplification by replacement: in time, and stepwise, bulky catalogs of empirical information (measurements, data, complex empirical models and rules) are replaced by much simpler summarizing statements (concepts, formulas, constitutive relations, principles, laws).

The simplest and most universal are the laws. The bulky and laborious are being replaced by the compact and the fast. In time, science optimizes and organizes itself in the same way as a river basin: toward configurations (links, connection) that provide better access, or easier flowing. The bulky measurements of pressure drop versus flow rate through round pipes and saturated porous media were rendered unnecessary by the formulas of Poiseuille and Darcy. The measurements of how things fall (faster and faster, and always from high to low) were rendered unnecessary by Galilei's principle and the second law of thermodynamics.

The hierarchy that science exhibited at every stage in the history of its development is an expression of its never ending struggle to redesign itself. Hierarchy means that measurements, ad-hoc assumptions and empirical models come in high numbers, above which the simple statements rise as sharp peaks. Both are needed, the numerous and the singular. One class of flows sustains the other. The many and unrelated heat engine builders of Cornwall and Scotland fed the imagination of one Sadi Carnot. In turn, Sadi Carnot's mental viewing (thermodynamics today) feeds the minds of contemporary and future builders of machines and atmospheric circulation models.

Science is this never ending process of generation of new configurations. Better flowing configurations replace existing configurations. The hands-on developers of empirical models and heat engines are numerous, like the hill slopes and the rivulets of a river basin. The principles of Galilei and Carnot are the big rivers, the Seine and the Danube.

Emerging today is a science of flow systems with configurations (Bejan and Lorente, 2004, 2005). A flow system has more than flow rate and dynamics, which are accounted for by principles such as mass conservation and Newton's second law of motion. A flow system has configuration (geometry) and freedom to morph. The "boundary conditions" that we assume routinely in order to solve the Navier-Stokes equations are in fact the big unknown: the configuration. 
Can the natural occurrence of flow configuration be reasoned on the basis of a single principle? In this review paper I show that the answer is yes, and that the principle is the constructal law (Sect. 1). The generation of flow configuration in time is a natural phenomenon, as natural as the one-way direction (irreversibility) of anything that flows.

Edited by: M. Sivapalan

\section{References}

Bejan, A.: Entropy Generation through Heat and Fluid Flow, Wiley, New York, 1982.

Bejan, A.: Heat Transfer, Wiley, New York, 1993.

Bejan, A.: Entropy Generation Minimization, CRC Press, Boca Raton, FL, 1996a.

Bejan, A.: Street network theory of organization in Nature, J. Adv. Transport., 30, 85-107, 1996b.

Bejan, A.: Constructal-theory network of conducting paths for cooling a heat generating volume, Int. J. Heat Mass Trans., 40, 799816, 1997a (published 1 November 1996).

Bejan, A.: Theory of organization in Nature: Pulsating physiological processes, Int. J. Heat Mass Trans., 40, 2097-2104, 1997 b.

Bejan, A.: Constructal tree network for fluid flow between a finitesize volume and one source or sink, Int. J. Thermal Sci., 36, 592604, 1997c.

Bejan, A.: Advanced Engineering Thermodynamics, 2nd ed., Wiley, New York, 1997d.

Bejan, A.: Shape and Structure, from Engineering to Nature, Cambridge University Press, Cambridge, UK, 2000.

Bejan, A.: Convection Heat Transfer, 3rd ed., Wiley, Hoboken, NJ, 2004.

Bejan, A.: Advanced Engineering Thermodynamics, 3rd ed., Wiley, Hoboken, NJ, 2006.

Bejan, A. and Errera, M. R.: Deterministic tree networks for river drainage basins, Fractals, 6, 245-261, 1998.

Bejan, A. and Gobin, D.: Constructal theory of droplet impact geometry, Int. J. Heat Mass Trans., 49, 2412-2419, 2006.

Bejan, A. and Lorente, S.: Thermodynamic formulation of the constructal law, Int. Mech. Eng. Congress and Expo, ASME Paper IMECE2003-41167, 2003.

Bejan, A. and Lorente, S.: The constructal law and the thermodynamics of flow systems with configuration, Int. J. Heat Mass Trans., 47, 3203-3214, 2004.

Bejan, A. and Lorente, S.: La Loi Constructale, L'Harmattan, Paris, 2005.

Bejan, A. and Marden, J. H.: Unifying constructal theory for scale effects in running, swimming and flying, J. Exp. Biol., 209, 238248, 2006.

Bejan, A. and Reis, A. H.: Thermodynamic optimization of global circulation and climate, Int. J. Energy Res., 29, 303-316, 2005.

Bejan, A., Dincer, I., Lorente, S., Miguel, A. F., and Reis, A. H.: Porous and Complex Flow Structures in Modern Technologies, Springer, New York, 2004.

Bejan, A., Lorente, S., Miguel, A. F., and Reis, A. H.: Constructal theory of distribution of river sizes, Sect. 13.5, in: Bejan, A.: Advanced Engineering Thermodynamics, 3rd ed., Wiley, Hoboken, NJ, 2006.
Carrigan, C. R.: Two-component magma transport and the origins of composite intrusions and lava flows, ch. 14 in: Magmatic Systems, edited by: Ryan, M. P., Academic Press, New York, 319353, 1994.

Carrigan, C. R. and Eichelberger, J. C.: Zoning of magmas by viscosity in volcanic conduits, Nature, 343, 248-251, 1990.

Chorley, R. J., Schumm, S. A., and Sugden, D. E.: Geomorphology, Methuen, London, 1984.

Dewar, R.: Information theory explanation of the fluctuation theorem, maximum entropy production and self-organized criticality in non-equilibrium stationary states, J. Phys. A: Math. Gen., 36, 631-641, 2003.

Hess, W. R.: Das Prinzip des kleinsten Kraftverbrauchs im Dienste hämodynamischer Forschung, Archiv. Anat. Physiol., 1913, see Weibel, 2000.

Kockman, N., Kiefer, T., Engler, M., and Woias, P.: Channel networks for optimal heat transfer and high throughput mixers, ECI Int. Conf. Heat Transfer and Fluid Flow in Microscale, Castelvecchio Pascoli, Italy, 25-30 September 2005.

Ledezma, G. A., Bejan, A., and Errera, M. R.: Constructal tree networks for heat transfer, J. Appl. Phys., 82, 89-100, 1997.

Leopold, L. B., Wolman, M. G., and Miller, J. P.: Fluvial Processes in Geomorphology, Freeman, San Francisco, 1964.

Lewins, J.: Bejan's constructal theory of equal potential distribution, Int. J. Heat Mass Trans., 46, 1541-1543, 2003.

Lin, C. A.: An extremal principle for a one-dimensional climate model, Geophys. Res. Lett., 9, 716-718, 1982.

Lorente, S. and Bejan, A.: Svelteness, freedom to morph, and constructal multi-scale flow structures, Int. J. Thermal Sci., 44, 1123-1130, 2005.

Lorentz, R. D., Lunine, J. I., McKay, C. P., and Withers, P. G.: Titan, Mars and Earth: entropy production by latitudinal heat transport, Geophys. Res. Lett., 25, 415-418, 2001.

Lundell, F., Thonon, B., and Gruss, J. A.: Constructal networks for efficient cooling/heating, 2nd Conference on Microchannels and Minichannels, Rochester, NY, 2004.

Malkus, W. V. R.: The heat transport and spectrum of turbulence, Prof. R. Soc. Ser. A., 225, 196-212, 1954.

Murray, C. D.: The physiological principle of minimal work, in the vascular system, and the cost of blood-volume, Proc. Acad. Nat. Sci., 12, 207-214, 1926.

Neagu, M. and Bejan, A.: Constructal-theory tree networks of "constant" thermal resistance, J. Appl. Phys., 86, 1136-1144, 1999.

Nield, D. A. and Bejan, A.: Convection in Porous Media, Springer, New York, 1992.

Nield, D. A. and Bejan, A.: Convection in Porous Media, 3rd ed., Springer, New York, 2006.

North, G. R.: Energy balance climate models, Rev. Geophys. Space Phys., 19, 91-121, 1981.

Paltridge, G. W.: Global dynamics and climate, Quart. J. Roy. Meteorol. Soc., 101, 475-484, 1975.

Parker, R. S.: Experimental study of drainage basin evolution and its hydrologic implications, Hydrology Paper 90, Colorado State University, Fort Collins, CO, 1977.

Poirier, H.: Une théorie explique l'intelligence de la nature, Science \& Vie, 1034, 44-63, 2003.

Reis, A. H. and Bejan, A.: Constructal theory of global circulation and climate, Int. J. Heat Mass Trans., 49, 1857-1875, 2006.

Reis, A. H., Miguel, A. F., and Bejan, A.: Constructal theory of par- 
ticle agglomeration and design of air-cleaning devices, J. Phys. D., 39, 2311-2318, 2006.

Rodriguez-Iturbe, I., Rinaldo, A., Rigon, R., Bras, R. L., Marani, A., and Ijjasz-Vasquez, E.: Energy dissipation, runoff production, and the three-dimensional structure of river basins, Water Resour. Res., 28, 1095-1103, 1992.

Rodriguez-Iturbe, I. and Rinaldo, A.: Fractal River Basins, Cambridge University Press, Cambridge, UK, 1997.

Rosa, R. N., Reis, A. H., and Miguel, A. F.: Bejan's Constructal Theory of Shape and Structure, Évora Geophysics Center, University of Évora, Portugal, 2004.

Scheidegger, A. E.: Theoretical Geomorphology, 2nd ed., SpringerVerlag, Berlin, 1970.
Swenson, R.: Emergent attractors and a law of maximum entropy production: foundations to a theory of general evolution, Syst. Res., 6, 187-197, 1989.

Thompson, D. A.: On Growth and Form, Cambridge University Press, Cambridge, UK, 1942.

Torre, N.: La Natura, vi svelo le formule della perfezione, La Macchina del Tempo, No. 1-2, Year 5, 36-46, 2004.

West, G. B. and Brown, J. H.: Constructing a theory of scaling and more, Physics Today, 20-21, July 2005.

West, G. B., Brown, J. H., and Enquist, B. J.: A general model for the origin of allometric scaling laws in biology, Science, 276, 122-126, 1997.

Whitfield, J.: Order out of chaos, Nature, 436, 905-907, 2005.

Weibel, E. R.: Symmorphosis, Harvard University Press, Cambridge, MA, 2000. 